\title{
Medical Emergencies in Dental Office
}

It gives me great pleasure to chalk out an Editorial for the terminal issue of 2013 Volume of Journal of International Oral Health (JIOH). Since the opportunity availed at the outset, a very serious title has stricken me which reminds of the domain I have been working on since past 6 years to discuss herewith. The expanse of "Medical Emergencies in Dental Office" what I wish to exclaim is the rubric which has the necessity of an exigent heed to the fraternity of dentistry today. I emphasize "today", the rationale being most of the populace is living an unhealthy life style due which the chances of unnoticed morbidity is aggrandized.

Due to this glorified state, the risk of potential complications for routine dental procedures also becomes alpine thus leaving the operator to be more capacitive to manage such intricacies. In the real front, such complexities do

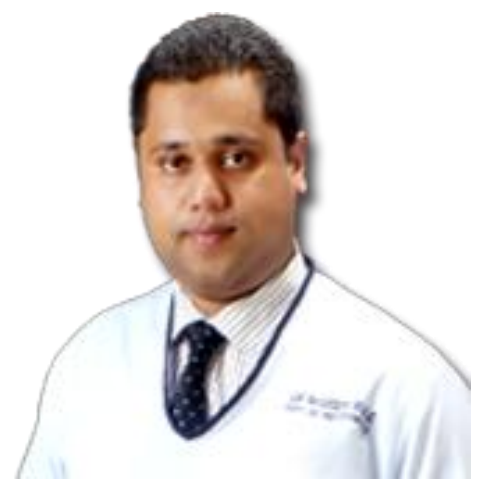

Dr. Mohammed Nadeem Ahmed Bijle

Executive Editor

Journal of International Oral Health not occur frequently for which most of the operators find themselves in a state of panic. Thus, if continuous drills of medical simulation are provided to the operators on a regular basis, the management of emergencies as afore-stated would not leave one in hysteria. It is also not expected for dental surgeons to be expert in emergency care as and when needed, but at least they must have the dexterity to recognize such emergent situations and assess the level of basic intervention needed to prevent further deterioration from the existing condition. This can be followed with definitive care as required depending upon the medical condition of the patient in jeopardy through seeking expert consultancy. However, such predictable outcomes are only possible if one is continuously in practice and updates oneself as per the current protocols. Thus, continuous renewal of knowledge and skills as per the recent protocols as suggested by regulatory authorizes viz., American Heart Association (AHA), American Society of Anesthesiologists (ASA), etc. in the field becomes the need of an hour.

I would like to redirect you to the portal of Highlights of AHA 2010 protocols delineating guidelines for CardioPulmonary Resuscitation (CPR) and Emergency Cardiovascular Care (ECC) where a special emphasis needs disposition on Basic Life Support (BLS) which I feel is the minimum necessity to be known by all health care providers including dental surgeons. Apart from possessing knowledge, a regular practice of the protocol also becomes a mandatory deed for the addressed populace. The necessary equipments and the associated drugs required to manage such emergencies should also be available in the office and rechecked on timely basis to determine its culmination where it would not prove useful rather can be fatal.

Thus, I have herewith provided with background answering the key questions with the topic related and would expect a reform from the members of the fraternity to follow the suggestions as outlined and make the same a regular practice.

Thanks \& Regards,

\section{Dr. Mohammed Nadeem Ahmed Bijle}

Executive Editor, Journal of International Oral Health, Lecturer, Department of Pedodontics \& Preventive Dentistry,

Dr. D. Y. Patil Vidyapeeth, Pune; Dr. D. Y. Patil Dental College \& Hospital, Pimpri, Pune,

Maharashtra. India.

E-mail: executive-editor@jioh.in 